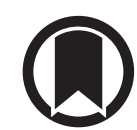

CrossMark

\title{
The global significance of PRISm: how data from low- and middle-income countries link physiology to inflammation
}

\author{
To the Editor:
}

We read with great interest the article by WiJNANT et al. [1] in a recent issue of European Respiratory Journal investigating the longitudinal outcomes of patients with preserved ratio impaired spirometry (PRISm) in the Rotterdam cohort. PRISm is a subject of increased investigation as it is associated with respiratory symptoms, cardiovascular disease, metabolic syndrome and, quite clearly in the recent study, increased mortality [1-3]. While there have been numerous studies examining outcomes related to PRISm in high-income countries (HICs), few studies have assessed the clinical significance of this spirometric finding in low- and middle-income countries (LMICs).

Globally, $85 \%$ of non-communicable disease deaths occur in LMICs. Of these, chronic respiratory disease is the fourth leading cause of death and the World Health Organization predicted the largest increase in global mortality to be attributed to these disorders [4]. As the prevalence of chronic respiratory disease in LMICs is increasing, it is clear that PRISm is one of the most common spirometry findings in LMICs $[5,6]$. Impairment on spirometry has been demonstrated to be as high as $60 \%$ among individuals with preserved forced expiratory volume in $1 \mathrm{~s}\left(\mathrm{FEV}_{1}\right) /$ forced expiratory volume (FVC) in the BOLD study and a more recent cross-sectional study in Malawi revealed 38.6\% of participants with PRISm [6]. Furthermore, there are unique risk factors that may lead to the development of this spirometric pattern in LMICs. WIINANT et al. [1] found that incident PRISm was significantly higher in patients with elevated body mass index (BMI) $(p<0.001$ and $p<0.010$ respectively); this finding was also present in the COPDGene cohort $[1,3]$. While obesity explains PRISm physiologically, its association with PRISm is not consistent. In the aforementioned Malawian and BOLD cohort, PRISm was paradoxically associated with lower BMI as well as poverty $[5,6]$. There have been even fewer longitudinal studies of PRISm in LMICs. One, a study in urban and rural Peru demonstrated increased lung function decline $\left(\mathrm{FEV}_{1}\right)$ among those with PRISm compared to normal spirometry [7], similar to the findings of Wijnant et al. [1]. It is worth noting, that high rates of PRISm could result from poorly performed spirometry, however, the studies mentioned conducted extensive training and ensured quality control checks on all spirometry data $[5,6,8]$.

How can we reconcile the very high rates of PRISM in LMICs if not associated with extra-thoracic restriction? One hypothesis of interest is predicated on the association of PRISm with ambient pollution and organic inhalational exposures. The rates of PRISM are higher in smokers, as noted in the COPDGene and MESA studies $[3,8]$. In LMICs, the rate of smoking remains much lower than that in HICs. However, biomass exposure and farming, which are prevalent through rural areas globally, have been strongly associated with impairment on spirometry in these settings. Additionally, the link between pollution and PRISm has been supported in numerous large cohorts in the USA and Europe [2]. Animal models further support this association, with links clearly identified between inhalational exposure, inflammation and impaired FVC [9]. All of these risk factors are far more prevalent in LMICs and may suggest a clue to the high prevalence in these settings.

So, what could be the common thread between the metabolic syndrome and inhalational exposure? A compelling association, described by WIJNANT et al. [1] and demonstrated in LMIC settings, is systemic inflammation. In Peru, individuals with PRISm were more likely to have glucose intolerance and have elevated high-sensitivity C-reactive protein independent of obesity and other comorbidities, indicating that a similar inflammatory pattern found in high-income settings may be at play [8]. While diabetes has been associated with PRISm in HICs, there is evidence that impairment on spirometry precedes glucose

@ERSpublications

Does the high rate and unique risk factors of preserved ratio impaired spirometry in low- and middleincome countries have something to teach us about the physiology of this highly prevalent pattern? http://bit.ly/2SsQjeG

Cite this article as: Jackson P, Siddharthan T. The global significance of PRISm: how data from low- and middle-income countries link physiology to inflammation. Eur Respir J 2020; 55: 2000184 [https://doi.org/ 10.1183/13993003.00184-2020]. 
intolerance. In the CARDIA study, individuals with higher fibrinogen experienced accelerated loss of lung function over time, though with a preserved ratio and a Swedish study reported similar findings with a range of circulatory inflammatory biomarkers $[10,11]$.

While compelling, the link between systemic inflammation and PRISm requires additional study. This will involve a commitment to establishing cohorts of PRISm patients in LMICs and ensuring that these patients obtain laboratory, radiological and detailed exposure assessments. In an ongoing project, our group at Johns Hopkins intend to evaluate PRISm patients from a large existing cohort in Kampala, Uganda with advanced pulmonary function testing and computed tomography scans. This study will allow comparisons of inhalational risk factors associated with this pattern and give additional longitudinal information about a LMIC cohort with high prevalence of PRISm.

Why does this matter in LMIC settings? With such a high prevalence of PRISm and clear evidence of increased mortality among patients with this physiological pattern, there may be a "time bomb" of patients that have thus far been overlooked. The increasing prevalence of spirometry in LMICs allows for increased recognition of this problem and the possibility for early intervention. All of these factors compel us to pursue further study of the PRISm phenotype, its associated risk factors and work to bridge the gap between PRISm patients in HICs and abroad.

Peter Jackson $\oplus^{1,2}$ and Trishul Siddharthan $\oplus^{1,2}$

${ }^{1}$ Division of Pulmonary and Critical Care, School of Medicine, Johns Hopkins University, Baltimore, MD, USA. ${ }^{2}$ Center for Global Non-Communicable Disease Research and Training, Johns Hopkins University, Baltimore, MD, USA.

Correspondence: Peter Jackson, Johns Hopkins University School of Medicine, Pulmonary Critical Care, 1830 E. Monument, 5th Floor, Baltimore, MD 21205-2105, USA. E-mail: pjacks35@jh.edu

Received: 28 Jan 2020 | Accepted after revision: 30 Jan 2020

Support statement: This work was funded by the Fogarty International Center (grant: 5108724). Funding information for this article has been deposited with the Crossref Funder Registry.

Conflict of interest: None declared.

\section{References}

1 Wijnant SRA, De Roos E, Kavousi M, et al. Trajectory and mortality of preserved ratio impaired spirometry: the Rotterdam Study. Eur Respir J 2020; 55: 1901217.

2 Godfrey MS, Jankowich MD. The vital capacity is vital: epidemiology and clinical significance of the restrictive spirometry pattern. Chest 2016; 149: 238-251.

3 Wan ES, Fortis S, Regan EA, et al. Longitudinal phenotypes and mortality in preserved ratio impaired spirometry in the COPDGene Study. Am J Respir Crit Care Med 2018; 198: 1397-1405.

4 World Health Organization. Global action plan for the prevention and control of noncommunicable diseases 2013-2020. Geneva, World Health Organization, 2013. Available from: http://apps.who.int/iris/bitstream/10665/ 94384/1/9789241506236_eng.pdf

5 Mannino DM, McBurnie MA, Tan W, et al. Restricted spirometry in the Burden of Lung Disease Study. Int J Tuberc Lung Dis 2012; 16: 1405-1411.

6 Meghji J, Nadeau G, Davis KJ, et al. Noncommunicable lung disease in sub-Saharan Africa. A community-based cross-sectional study of adults in urban Malawi. Am J Respir Crit Care Med 2016; 194: 67-76.

7 Siddharthan T, Grigsby $\mathrm{M}$, Miele $\mathrm{CH}$, et al. Prevalence and risk factors of restrictive spirometry in a cohort of Peruvian adults. Int J Tuberc Lung Dis 2017; 21: 1062-1068.

8 Armstrong HF, Lovasi GS, Soliman EZ, et al. Lung function, percent emphysema, and QT duration: the Multi-Ethnic Study of Atherosclerosis (MESA) lung study. Respir Med 2017; 123: 1-7.

9 de Barros Mendes Lopes T, Groth EE, Veras M, et al. Pre- and postnatal exposure of mice to concentrated urban PM2.5 decreases the number of alveoli and leads to altered lung function at an early stage of life. Environ Pollut 2018; 241: 511-520.

10 Thyagarajan B, Jacobs DR, Apostol GG, et al. Plasma fibrinogen and lung function: the CARDIA Study. Int $J$ Epidemiol 2006; 35: 1001-1008.

11 Engström G, Lind P, Hedblad B, et al. Lung function and cardiovascular risk: relationship with inflammationsensitive plasma proteins. Circulation 2002; 106: 2555-2560.

Copyright (CERS 2020

From the authors:

In a recent paper published in the European Respiratory Journal, we studied the characteristics, trajectory and mortality of subjects with Preserved Ratio Impaired Spirometry (PRISm) in the Rotterdam Study, a prospective population-based cohort study in the Netherlands, a high-income country (HIC) [1]. In an elegant letter to the editor, P. Jackson and T. Siddharthan correctly argue that clinical research on PRISm 
TABLE 1 Systemic inflammatory markers in subjects with normal spirometry, PRISm or COPD in the Rotterdam Study

\begin{tabular}{lccc} 
Rotterdam Study (HIC) & Normal spirometry & PRISm & COPD \\
\hline Subjects $\mathbf{n}$ & 4185 & 387 & 915 \\
hs-CRP & $1.26 \pm 3.09$ & $1.57 \pm 2.00^{\#}$ & $1.54 \pm 2.80^{*}$ \\
WBC & $6.92 \pm 1.89$ & $7.46 \pm 2.04^{*}$ & $7.64 \pm 2.40^{*}$ \\
Granulocyte count & $4.09 \pm 131$ & $4.65 \pm 1.67^{*}$ & $4.73 \pm 1.62^{*}$ \\
Granulocyte \% & $58.71 \pm 8.60$ & $61.56 \pm 8.30^{*}$ & $61.59 \pm 8.82^{*}$ \\
Lymphocyte count & $2.38 \pm 1.05$ & $2.35 \pm 0.74$ & $2.42 \pm 1.42$ \\
Lymphocyte \% & $34.61 \pm 8.12$ & $32.04 \pm 7.92^{*}$ & $31.87 \pm 8.18^{*}$ \\
Neutrophil to lymphocyte ratio & $1.87 \pm 0.82$ & $2.12 \pm 0.87^{*}$ & $2.16 \pm 1.00^{*}$ \\
Thrombocyte count & $266.38 \pm 66.32$ & $269.01 \pm 70.22$ & $273.29 \pm 70.58^{*}$ \\
Systemic immune inflammation index & $497.40 \pm 264.52$ & $577.96 \pm 318.83^{*}$ & $592.41 \pm 334.03^{*}$ \\
\hline
\end{tabular}

Data are presented as $\mathrm{n}$ or mean \pm SD. hs-CRP: high-sensitive C-reactive protein; PRISm: preserved ratio impaired spirometry; WBC: white blood cell count. The systemic immune inflammation index is the granulocyte count multiplied by the thrombocyte count, divided by lymphocyte count. *: $p<0.05$ and \# : $p<0.10$ compared to subjects with normal spirometry. There are 396 missing values for hs-CRP. hs-CRP was measured on average $10.03 \pm 4.90$ years prior to spirometry. Other inflammatory markers were measured at the time of spirometry.

has been limited so far in its potential to grasp a representative sample of the world population, with marked underrepresentation of studies in low- and middle-income countries (LMICs). Despite potential differences in risk factors, they suggest that systemic inflammation plays a key role in the pathogenesis of PRISm in both HIC and LMICs. In the Rotterdam Study, we showed an increased white blood cell count in subjects with PRISm as compared to subjects with normal spirometry [1]. In addition, subjects with PRISm have higher serum levels of high-sensitive C-reactive protein, a higher granulocyte count, a higher granulocyte to lymphocyte ratio and a higher systemic immune inflammation index (table 1).

P. Jackson and T. Siddharthan state that while extra-thoracic pulmonary restriction, through for example obesity or cardiovascular diseases (encompassing heart failure and/or ischaemic heart disease), is associated with PRISm in HICs, air pollution and organic inhalational exposures are more likely associated with PRISm in LMICs. Since PRISm is a heterogenous syndrome encompassing multiple subgroups, we propose that a spectrum of mechanisms and risk factors contribute to varying degrees to the pathogenesis of PRISm in different populations. With globalisation comes modernisation and westernisation of lifestyles, also in LMICs. Therefore, we argue that also in LMICs, obesity might play an important role in a subgroup of subjects with PRISm. Abdominal obesity may indeed compromise lung volumes through the mechanical effects of truncal obesity and/or the metabolic effects of adipose tissue. To what extent either mechanical or metabolic effects of obesity lead to PRISm is unknown, though the postulated hypothesis by P. Jackson and T. Siddharthan of systemic inflammation linking PRISm in both HIC and LMICs suggests a relatively higher importance of the metabolic effects.

Another, but underappreciated, risk factor for PRISm is impaired lung growth [2]. Especially, but not exclusively, in LMICs, environmental factors such as air pollution, smoking, malnutrition and respiratory infections may limit optimal lung growth during fetal life, childhood and adolescence. Indeed, a history of pneumonia or pleurisy in childhood has been shown to increase the prevalence of PRISm later in life in a HIC [3], with likely larger estimates in LMICs due to higher rates and severity of pulmonary infections (including tuberculosis). Also, a well-balanced nutrition in childhood and adolescence is important for optimal lung growth. For example, in case of endemic deficiency of vitamin A, supplementation of vitamin A in pregnant women was associated with increased lung function in their offspring [4]. Infection, malnutrition and exposure to air pollutants (e.g. organic compounds and particulate matter) likely all play a role in limiting lung function growth and hence, in increasing the prevalence of PRISm in a Malawian study [5], whereas the same risk factors, as well as obesity, may contribute to PRISm in other countries, yet with varying degrees of importance for each specific risk factor and setting.

Lastly, clinical phenotypes (including spirometric measures, such as forced vital capacity (FVC)) are the result of interactions between multiple environmental factors and an individual's unique genome. Indeed, genome-wide association studies have identified multiple genetic variants (mostly single-nucleotide polymorphisms, but also deletions, insertions and copy number variants) associated with FVC in individuals of European ancestry. These genetic variants have been implicated in lung development, repair 
and response to injury [6]. It is unclear whether genetic susceptibility differs in individuals from non-European ancestry, predisposing to a larger extent of impaired lung growth and PRISm in LMICs.

To conclude, we agree that there is a need for more studies to disentangle risk factors, mechanisms and trajectories of PRISm, especially in LMICs. PRISm has been neglected in many research papers in the past, and as a result is often ignored in clinical practice, despite its unfavourable outcomes. One of the main conclusions of our paper was the heterogeneity of PRISm, a message we need to reinforce: subjects with PRISm represent a heterogeneous population encompassing distinct phenotypes with distinct risk factors. Upon identification of PRISm on spirometry, more efforts towards understanding the individual's specific pathogenesis of PRISm is warranted in order to improve therapeutic approaches. The association of systemic inflammation with PRISm should be further disentangled, as well as replicated in studies from LMICs. Moreover, the relative importance of systemic inflammation in the link between obesity and PRISm should be further clarified. Finally, through large-scale epidemiological research in LMICs, new risk factors for PRISm can be discovered, leading towards a better understanding of this peculiar spirometric phenotype. Prospective studies are warranted to elucidate factors leading to impaired lung growth in childhood and their effects on lung function later in life.

@ERSpublications

Subjects with PRISm represent a heterogeneous population encompassing distinct phenotypes with distinct risk factors. A spectrum of mechanisms and risk factors contribute to varying degrees to the pathogenesis of PRISm in different populations. http://bit.ly/2TkHJjK

Cite this article as: Wijnant SRA, Lahousse L, Brusselle GG. The global significance of PRISm: how data from low- and middle-income countries link physiology to inflammation. Eur Respir J 2020; 55: 2000354 [https://doi.org/10.1183/13993003.00354-2020].

Sara R.A. Wijnant $\oplus^{1,2,3}$, Lies Lahousse $\oplus^{2,3}$ and Guy G. Brusselle $\odot^{1,2,4}$

${ }^{1}$ Dept of Respiratory Diseases, Ghent University Hospital, Ghent, Belgium. ${ }^{2}$ Dept of Epidemiology, Erasmus Medical Center, Rotterdam, The Netherlands. ${ }^{3}$ Dept of Bioanalysis, Faculty of Pharmaceutical Sciences, Ghent University, Ghent, Belgium. ${ }^{4}$ Dept of Respiratory Diseases, Erasmus Medical Center, Rotterdam, The Netherlands.

Correspondence: Guy G. Brusselle, Ghent University Hospital, Dept of Respiratory Diseases, 7K12 IE, De Pintelaan 185, Ghent, B-9000, Belgium. E-mail: guy.brusselle@UGent.be

Received: 18 Feb 2020 | Accepted after revision: 19 Feb 2020

Conflict of interest: S.R.A. Wijnant reports grants from GlaxoSmithKline, outside the submitted work. L. Lahousse reports grants from AstraZeneca and Chiesi (both awards), and expert consultation for Boehringer Ingelheim GmbH and Novartis, outside the submitted work. G.G. Brusselle reports personal fees for advisory board work and lectures from AstraZeneca, Boehringer Ingelheim, Chiesi, GlaxoSmithKline, Novartis and Teva, personal fees for advisory board work from Sanofi, outside the submitted work.

\section{References}

1 Wijnant SRA, Roos E, Kavousi M, et al. Trajectory and mortality of preserved ratio impaired spirometry: the Rotterdam Study. Eur Respir J 2020; 55: 1901217.

2 Agustí A, Hogg JC. Update on the pathogenesis of chronic obstructive pulmonary disease. N Engl J Med2019; 381: $1248-1256$.

3 Perret JL, Lodge CJ, Lowe AJ, et al. Childhood pneumonia, pleurisy and lung function: a cohort study from the first to sixth decade of life. Thorax 2020; 75: 28-37.

4 Checkley W, West KP, Wise RA, et al. Maternal vitamin A supplementation and lung function in offspring. $N$ Engl J Med 2010; 362: 1784-1794.

5 Meghji J, Nadeau G, Davis KJ, et al. Noncommunicable lung disease in sub-Saharan Africa. A community-based cross-sectional study of adults in urban Malawi. Am J Respir Crit Care Med 2016; 194: 67-76.

6 Loth DW, Artigas MS, Gharib SA, et al. Genome-wide association analysis identifies six new loci associated with forced vital capacity. Nat Genet 2014; 46: 669-677. 